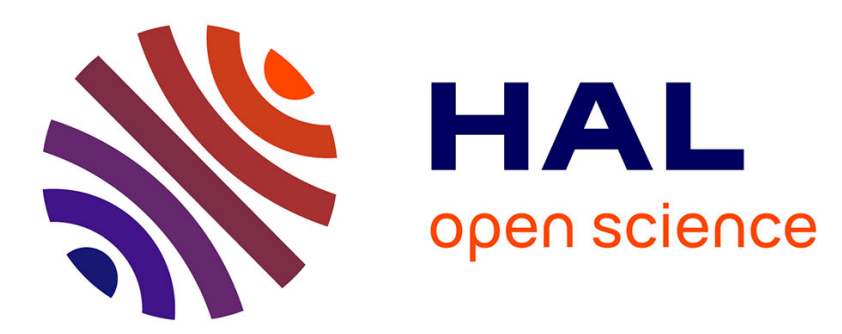

\title{
Effet Hall de chlorures et d'iodures en solutions diluées dans le méthanol et dans l'eau
}

\author{
M. Abbes, R. Gérard, P. Gérard, M. Meton, E.J. Picard
}

\section{To cite this version:}

M. Abbes, R. Gérard, P. Gérard, M. Meton, E.J. Picard. Effet Hall de chlorures et d'iodures en solutions diluées dans le méthanol et dans l'eau. Journal de Physique Lettres, 1982, 43 (5), pp.127132. 10.1051/jphyslet:01982004305012700 . jpa-00232020

\section{HAL Id: jpa-00232020 https://hal.science/jpa-00232020}

Submitted on 1 Jan 1982

HAL is a multi-disciplinary open access archive for the deposit and dissemination of scientific research documents, whether they are published or not. The documents may come from teaching and research institutions in France or abroad, or from public or private research centers.
L'archive ouverte pluridisciplinaire HAL, est destinée au dépôt et à la diffusion de documents scientifiques de niveau recherche, publiés ou non, émanant des établissements d'enseignement et de recherche français ou étrangers, des laboratoires publics ou privés. 


\title{
LE JOURNAL DE PHYSIQUE-LETTRES
}

J. Physique - LETTRES 43 (1982) L-127 - L-132

1er MARS 1982, PAGE L-127

Classification

Physics Abstracts

82.90

\section{Effet Hall de chlorures et d'iodures en solutions diluées dans le méthanol et dans l'eau}

\author{
M. Abbes, R. Gérard, P. Gérard, M. Meton et E. J. Picard \\ Laboratoire de Recherche sur l'Effet Hall, Université Pierre-et-Marie-Curie, \\ U.E.R. Application de la Physique, Tour 46, 4, place Jussieu, 75230 Paris Cedex 05, France
}

(Reçu le 19 octobre 1981, accepté le 13 janvier 1982)

Résumé. - Nous présentons les premières mesures d'Effet Hall de solutions électrolytiques diluées de chlorures et d'iodures ayant pour solvant l'alcool méthylique. Nous comparons les résultats ainsi obtenus avec ceux que l'on obtient dans les solutions aqueuses des mêmes sels.

\begin{abstract}
This letter presents the first Hall Effect measurements of dilute electrolytic solutions of chloride and iodide salts in methylic alcohol. We compare these results with those obtained for the same salts in water.
\end{abstract}

1. Introduction. - Nos premières tentatives pour mesurer l'Effet Hall dans des solutions électrolytiques ayant le méthanol pour solvant se sont soldées par un échec. Etanches aux solutions aqueuses, nos cellules ne l'étaient pas aux solutions alcooliques plus fluides. En outre, nous avons pu constater que la solution alcoolique s'échappait par un ou plusieurs des orifices de remplissage et mouillait la face supérieure externe de la cellule, manifestant de manière amplifiée, un phénomène pratiquement imperceptible avec l'eau. Les mesures dans le méthanol étaient impossibles, alors que les mesures dans l'eau, effectuées jusqu'alors, avaient parfois montré une dispersion gênante dont nous avions enfin l'explication : un film très mịnce de solution débordant de la cellule favorisait probablement des fuites électriques plus ou moins importantes.

La réalisation d'un nouveau porte-cellule de conception différente nous a permis de venir à bout d'une partie des difficultés : une nouvelle disposition des électrodes en platine, l'utilisation de joints en téflon ont assuré l'étanchéité des cellules. La mise au point d'une nouvelle méthode de remplissage - remplissage plus progressif évitant tout débordement de liquide, et remplissage incomplet laissant subsister des bulles d'air isolant la surface libre de l'électrolyte des orifices 


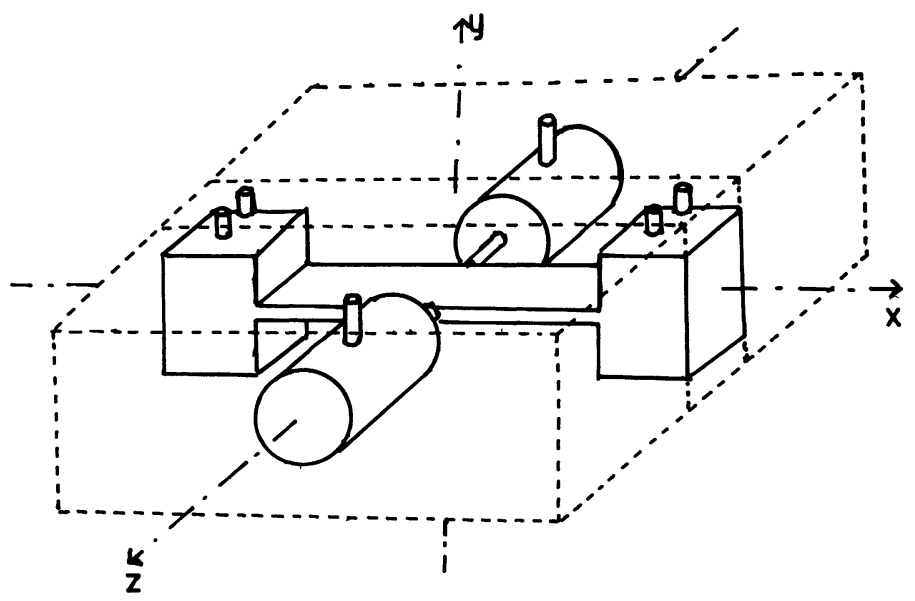

Fig. 1. - Schéma de la cellule de Hall. En trait continu le contour du volume réservé au liquide électrolytique avec les six orifices de remplissage. En trait discontinu les limites externes de la cellule.

[Hall cell diagram. Full line delimits the volume occupied by the electrolyte. Note the six filling orifices. Dotted line delimits the outside cell boundary.]

capillaires de remplissage (voir Fig. 1) - ont interdit toute fuite de liquide et permis des mesures sûres et reproductibles dans le méthanol et dans l'eau.

Ayant ainsi pu mesurer l'Effet Hall de chlorures et d'iodures dans le méthanol, nous avons repris l'étude des chlorures et des iodures effectuée antérieurement dans l'eau [1,2] et nous l'avons étendue à de nouveaux ions. Cela nous a permis de recouper les premiers résultats et de les préciser.

Nous donnerons d'abord quelques précisions sur l'aspect expérimental de la méthode utilisée et nous rappellerons quelques définitions et formules utiles; nous présenterons ensuite les résultats des mesures et des calculs; la comparaison des résultats obtenus dans l'alcool méthylique et dans l'eau nous permettra enfin de conclure, sous réserve de confirmation ultérieure, à une différence de comportement dynamique des ions dans les deux solvants.

2. Aspect expérimental. - Nous utilisons, rappelons-le, la méthode de la double fréquence : un courant alternatif de $243,75 \mathrm{~Hz}$ et un champ alternatif de $75 \mathrm{~Hz}$ engendrent une tension de Hall transversale qui comporte deux composantes sinusoïdales (fréquence différence de $168,75 \mathrm{~Hz}$ et fréquence somme de $318,75 \mathrm{~Hz}$ ). On trouvera l'organigramme complet de l'appareillage expérimental dans une précédente publication [3]. La cellule qui contient la lame électrolytique étudiée est réalisée à l'aide de quatre blocs de silice pure polis optiquement en adhésion moléculaire (voir Fig. 1); le courant de commande circule suivant $\mathrm{Ox}$, l'induction magnétique est uniforme et parallèle à $\mathrm{O} y$, la tension de Hall est mesurée suivant $\mathrm{O} z$. Les électrodes en platine sont planes et appliquées sur les quatre faces externes latérales de la cellule; des joints en téflon assurent l'étanchéité.

Les mesures ont été faites avec une cellule d'épaisseur $d=0,79 \mathrm{~mm}$ (la lame parallélépipédique au centre de la cuve a une largeur de $8 \mathrm{~mm}$ et une longueur de $3,5 \mathrm{~cm}$ ). Toutes les solutions étudiées ont la même concentration $c$ égale à $5 \times 10^{-3}$ éq. $1^{-1}$.

Le courant de commande $I_{\text {eff }}$ est fixé à $1,5 \mathrm{~mA}$; le champ magnétique $B_{\text {eff }}$ est maintenu à $0,2 \mathrm{~T}$.

Au départ la température des solutions est de 19 à $20^{\circ} \mathrm{C}$; elle s'élève en cours d'expérience et peut atteindre 24 à $25^{\circ} \mathrm{C}$ à la fin de celle-ci. Cette élévation de température est liée à la dissipation par effet Joule. 
Chaque valeur de tension de Hall mesurée $V_{\text {eff }}$ est une valeur moyenne sur au moins trois expériences portant sur des lames d'électrolyte différentes.

3. Résultats. - Les mesures de tension de Hall ont été effectuées sur 14 solutions aqueuses et 11 solutions alcooliques de chlorures et d'iodures de 7 cations monovalents. L'erreur absolue des mesures est comprise entre 5 et $11 \mathrm{nV}$.

De la relation $V_{\text {eff }}=R_{\mathrm{H}} I_{\text {eff }} B_{\text {eff }} / d \sqrt{2}$ nous tirons la valeur de la constante de Hall $R_{\mathrm{H}}$ qui est liée aux conductivités équivalentes ioniques $\lambda_{+}$et $\lambda_{-}$du cation et de l'anion par la relation :

$$
R_{\mathrm{H}}=\frac{1}{n|e|} \frac{\lambda_{+}^{2} h_{+}-\lambda_{-}^{2} h_{-}}{\left(\lambda_{+}+\left|\lambda_{-}\right|\right)^{2}}=\frac{1}{n|e|} \frac{\lambda_{+}^{2} h_{+}-\lambda_{-}^{2} h_{-}}{\Lambda^{2}}=\frac{1}{n|e|} h
$$

où $n$ est le nombre de porteurs d'une espèce par $\mathrm{m}^{3}\left(n=c \times 10^{3} .6,02 \times 10^{23}\right) ; R_{\mathrm{H}}$ s'exprime alors en $\mathrm{m}^{3} \cdot \mathrm{C}^{-1}$.

$h_{+}$et $h_{-}$sont les nombres de Hall ioniques du cation et de l'anion égaux par définition à

$$
h_{+}=\lambda_{+}^{\mathrm{m}} / \lambda_{+} \quad \text { et } h_{-}=\lambda_{-}^{\mathrm{m}} / \lambda_{-}
$$

$\lambda_{+}^{m}$ et $\lambda_{-}^{m}$ étant les conductivités ioniques magnétiques. $h$ est le nombre de Hall de l'électrolyte égal par définition à :

$$
h=\frac{\lambda_{+}^{2} h_{+}-\lambda_{-}^{2} h_{-}}{\left(\lambda_{+}+\left|\lambda_{-}\right|\right)^{2}} .
$$

A partir de l'équation (1) nous calculons les nombres de Hall $h$, puis nous formons les produits $\Lambda^{2} h$ pour les différents électrolytes. Si les relations ci-dessus sont vérifiées expérimentalement la différence :

$$
\left(\Lambda^{2} h\right)_{\mathrm{X}^{+} \mathrm{Cl}^{-}}-\left(\Lambda^{2} h\right)_{\mathrm{X}^{+1^{-}}}=\lambda_{1^{-}}^{2} h_{\mathrm{I}^{-}}-\lambda_{\mathrm{Cl}^{-}}^{2} h_{\mathrm{Cl}^{-}}
$$

doit être indépendante du cation $\mathrm{X}^{+}$.

A dilution infinie la relation (4) s'écrit :

$$
\left(\Lambda_{0}^{2} h_{0}\right)_{\mathrm{X}^{+} \mathrm{Cl}^{-}}-\left(\Lambda_{0}^{2} h_{0}\right)_{\mathrm{X}^{+\mathrm{I}^{-}}}=\lambda_{\mathrm{OI}^{-}}^{2} h_{\mathrm{OI}^{-}}-\lambda_{0 \mathrm{Cl}^{-}}^{2} h_{0 \mathrm{Cl} 1^{-}}
$$

Dans une précédente publication [3] nous avons montré, au moins pour trois solutions aqueuses, que le nombre de Hall d'une solution ne varie pas de façon appréciable dans un grand domaine de concentration. Nous avons en outre constaté que des variations de température de $5^{\circ} \mathrm{C}$ n'avaient pas d'influence mesurable sur la tension de Hall. C'est la raison pour laquelle nous avons utilisé la relation (4 bis) au lieu de la relation (4) pour nos calculs. Nous avons adopté les valeurs des conductivités équivalentes limites ioniques $\lambda_{0}$ à $25^{\circ} \mathrm{C}$ que l'on trouve dans la littérature [4]; au lieu des $h_{0}$, nombres de Hall limite, nous avons pris les valeurs des nombres de Hall $h$ mesurés sur les solutions de concentration $5 \times 10^{-3}$ éq. $1^{-1}$ à $20-24^{\circ} \mathrm{C}$. Nous n'avons, en outre, pas tenu compte des effets possibles de l'association, effets non négligeables dans le cas de quelques iodures en solution alcoolique, car nous avons estimé que ces effets restent négligeables devant les erreurs de mesure.

Les tableaux I et II montrent que les différences $\left(\Lambda_{0}^{2} h\right)_{\mathbf{X}^{+} \mathrm{Cl}^{-}}-\left(\Lambda_{0}^{2} h\right)_{\mathrm{X}^{+} \mathrm{I}^{-}}$sont constantes dans l'eau et dans le méthanol.

Pour les solutions aqueuses

$$
\lambda_{0 I^{-}}^{2} h_{1^{-}}-\lambda_{0 \mathrm{Cl}^{-}}^{2} h_{\mathrm{Cl}^{-}} \simeq 250 \text {. }
$$


Tableau I. - Résultats des mesures en solutions aqueuses.

[Measurement results in aqueous solutions.]

\begin{tabular}{|c|c|c|c|c|c|c|c|}
\hline & $\begin{array}{l}\mathrm{Bu}{ }_{4} \mathrm{~N}^{+} \\
\left(\lambda_{0}^{4}=19,31\right) \\
h_{+}=1 \\
h^{\prime}{ }_{+}=1,(1) \\
h^{\prime \prime}{ }_{+}=0,6(7)\end{array}$ & $\begin{array}{l}\mathrm{Et}_{4} \mathrm{~N}^{+} \\
(32,22) \\
0,8(3) \\
0,8(7) \\
0,7(1)\end{array}$ & $\begin{array}{c}\mathrm{Me}_{4} \mathrm{~N}^{+} \\
(44,42) \\
0,7(6) \\
0,7(8) \\
0,7(0) \\
\end{array}$ & $\begin{array}{l}\mathrm{Cs}^{+} \\
(77,29) \\
0,6(9) \\
0,7(0) \\
0,6(7) \\
\end{array}$ & $\begin{array}{c}x^{+} \\
(73,55) \\
0,7(0) \\
0,7(1) \\
0,6(7)\end{array}$ & $\begin{array}{c}\mathrm{Na}^{+} \\
(50,20) \\
0,7(5) \\
0,7(7) \\
0,7(0)\end{array}$ & $\begin{array}{c}21^{+} \\
(38,66) \\
0,8(3) \\
0,8(8) \\
0,7(5)\end{array}$ \\
\hline $\begin{array}{c}c 1^{-}\left(\lambda_{0}^{-}=76,39\right) \\
h_{-}=0,5(4), h_{-}^{\prime}=0,5(5), h_{-}^{n}=0,5(2)\end{array}$ & $\begin{array}{l}-169 \pm 11 \\
-6,29 \\
-0,304 \\
-2780 \pm 180\end{array}$ & $\begin{array}{l}-107 \pm 6 \\
-3,98 \\
-0,192 \\
-2270 \pm 130\end{array}$ & $\begin{array}{l}-64 \pm 6 \\
-2,4 \\
-0,115 \\
-1680 \pm 160\end{array}$ & $\begin{array}{l}+23 \pm 6 \\
+0,86 \\
+0,041 \\
+980 \pm 250\end{array}$ & $\begin{array}{l}+16 \pm 7 \\
+0,60 \\
+0,029 \\
+650 \pm 280\end{array}$ & $\begin{array}{l}-42 \pm 7 \\
-1,56 \\
-0,075 \\
-1210 \pm 200\end{array}$ & $\begin{array}{l}-80 \pm 7 \\
-3,0 \\
-0,144 \\
-1900 \pm 170\end{array}$ \\
\hline $\begin{aligned} I^{-} & (76,98) \\
0,5(7) & ; 0,5(9), 0,5(5)\end{aligned}$ & $\begin{array}{l}-180 \pm 7 \\
-6,70 \\
-0,323 \\
-3000 \pm 150\end{array}$ & $\begin{array}{l}-118 \pm 7 \\
-4,39 \\
-0,212 \\
-2530 \pm 150 \\
\end{array}$ & $\mid \begin{array}{l}-70 \pm 7 \\
-2,6 \\
-0,126 \\
-1850 \pm 180\end{array}$ & $\begin{array}{l}+18 \pm 5 \\
+0,67 \\
+0,032 \\
+770 \pm 200\end{array}$ & $\begin{array}{l}+9 \pm 7 \\
+0,34 \\
+0,016 \\
+370 \pm 300\end{array}$ & $\begin{array}{l}-53 \pm 7 \\
-1,97 \\
-0,095 \\
-1540 \pm 200\end{array}$ & $\begin{array}{l}-90 \pm 6 \\
-3,4 \\
-0,162 \\
-2160 \pm 140 \\
\end{array}$ \\
\hline$\lambda_{O \mathrm{II}}^{2} \mathrm{I}-\lambda_{O \mathrm{OCl}}^{2} \mathrm{~h} \mathrm{Cl}$ & $220 \pm 330$ & $260 \pm 280$ & $170 \pm 340$ & $210 \pm 450$ & $280 \pm 580$ & $330 \pm 400$ & $260 \pm 310$ \\
\hline $\begin{array}{l}\text { C16 } \\
\text { Key }\end{array}$ & \multicolumn{7}{|c|}{ 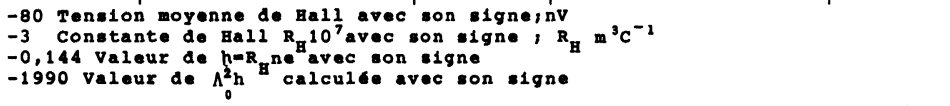 } \\
\hline
\end{tabular}

Tableau II. - Résultats des mesures en solutions alcooliques.

[Measurement results in alcoholic solutions.]

\begin{tabular}{|c|c|c|c|c|c|c|c|}
\hline & $\mid \begin{array}{l}\mathrm{Bu}_{4} \mathrm{~N}^{+} \\
\left(\mathrm{x}_{0}^{+}=38,94\right) \\
\mathrm{h}_{+}=1 \\
\mathrm{~h}^{+}{ }_{+}=0,8(8) \\
\mathrm{h}_{+}=0,6(4)\end{array}$ & $\mid \begin{array}{l}\mathrm{Et} 4^{\mathrm{N}^{+}} \\
(60,50) \\
0,8(8) \\
0,8(3) \\
0,7(3)\end{array}$ & $\begin{array}{c}\operatorname{Met}_{4} \mathrm{~N}^{+} \\
(68,73) \\
0,8(2) \\
0,7(9) \\
0,7(1)\end{array}$ & $\begin{array}{l}\mathrm{Cs}^{+} \\
(60,83) \\
0,7(5) \\
0,7 \\
0,6(0)\end{array}$ & $\begin{array}{c}\mathrm{x}^{+} \\
(52,44) \\
0,7(9) \\
0,7(0) \\
0,5(6)\end{array}$ & $\begin{array}{c}\mathrm{Na}^{+} \\
(45,17) \\
0,9(3) \\
0,7(1) \\
0,6(6)\end{array}$ & $\begin{array}{c}\mathrm{L1}^{+} \\
(39,55) \\
1,(1) \\
0,8(4) \\
0,7(6)\end{array}$ \\
\hline $\begin{array}{c}c 1^{-}\left(\lambda_{0}^{-}=52,36\right) \\
h_{-}=0,8(0), h_{-}^{\prime}=0,7(3), h_{-}=0,6\end{array}$ & & & $\begin{array}{l}+63 \pm 6 \\
+2,37 \\
+0,113 \\
+1660 \pm 160\end{array}$ & $\begin{array}{l}+28 \pm 6 \\
+1,04 \\
+0,053 \\
+640 \pm 140\end{array}$ & \begin{tabular}{|l}
$-2 \pm 8$ \\
$-0,07$ \\
$-0,004$ \\
$-40 \pm 160$
\end{tabular} & \begin{tabular}{|l|}
$-19 \pm 8$ \\
$-0,71$ \\
$-0,034$ \\
$-320 \pm 140$
\end{tabular} & $\begin{array}{l}-30 \pm 10 \\
-1,12 \\
-0,054 \\
-460 \pm 150\end{array}$ \\
\hline $\begin{array}{cl}I^{-} & (62,78) \\
0,7(3) & , 0,6(9), 0,6\end{array}$ & $\begin{array}{l}-74 \pm 7 \\
-2,76 \\
-0,133 \\
-1.380 \pm 130 \\
\end{array}$ & $\begin{array}{l}+12 \pm 6 \\
+0,45 \\
+0,022 \\
+330 \pm 160 \\
\end{array}$ & $\begin{array}{l}+33 \pm 6 \\
+1,23 \\
+0,059 \\
+1030 \pm 190 \\
\end{array}$ & $\begin{array}{l}-7 \pm 6 \\
-0,26 \\
-0,013 \\
-190 \pm 160 \\
\end{array}$ & $\begin{array}{l}-32 \pm 8 \\
-1,19 \\
-0,057 \\
-760 \pm 190 \\
\end{array}$ & $\begin{array}{l}-47 \pm 7 \\
-1,75 \\
-0,084 \\
-980 \pm 150 \\
\end{array}$ & \\
\hline$\lambda_{O I}^{2}{ }^{h}-\lambda_{O C 1}^{2}{ }^{h} \mathrm{Cl}$ & & & 620350 & 830300 & $\begin{array}{l}720 \quad 350\end{array}$ & $\begin{array}{ll}660 & 290\end{array}$ & \\
\hline $\begin{array}{l}\text { Cle } \\
\text { Xey }\end{array}$ & \multicolumn{7}{|c|}{ 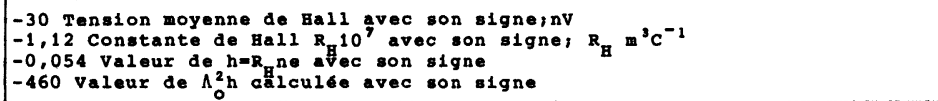 } \\
\hline
\end{tabular}

Les conductibilités de l'ion chlorure et de l'ion iodure étant très voisines, la relation (5) donne un ordre de grandeur de la différence $h_{1^{-}}-h_{\mathrm{Cl}^{-}} \simeq 4 \times 10^{-2}$.

Pour les solutions alcooliques

$$
\lambda_{01^{-}}^{2} h_{1^{-}}-\lambda_{0 \mathrm{Cl}}^{2} h_{\mathrm{Cl}^{-}} \simeq 710 .
$$

Les conductibilités de l'ion chlorure et de l'ion iodure sont, cette fois, très différentes, et si on admet que pour ce solvant, comme pour l'eau, $h_{\mathrm{I}^{-}} \simeq h_{\mathrm{Cl}^{-}}$, on obtient l'ordre de grandeur : $h_{1^{-}} \simeq h_{\mathrm{Cl}^{-}} \simeq 0,6$.

Dans une publication récente, J. B. Hubbart et P. G. Wolynes [5] ont calculé les forces électrohydrodynamiques qui s'exercent sur un ion en mouvement dans un liquide polarisable en 
présence d'un champ magnétique. Le résultat obtenu est indépendant de la taille, de la charge de l'ion et de la viscosité du solvant : la force de Lorentz est diminuée dans tous les cas d'environ $30 \%$. En d'autres termes, suivant cette théorie, les nombres de Hall ioniques seraient tous égaux à 0,7 .

Le tableau I, qui donne des tensions de Hall très nettement positives pour $\mathrm{K}^{+} \mathrm{Cl}^{-} \mathrm{ou} \mathrm{Cs}^{+} \mathrm{I}^{-}$, montre, qu'en solution aqueuse, les nombres de Hall ioniques sont, au moins, fonction du signe de la charge.

On sait que pour obtenir un ensemble de nombres de Hall ioniques dans l'eau et dans le méthanol, il faut faire une hypothèse concernant le nombre de Hall d'un ion. Jusqu'ici, nous avons admis que $h_{\mathrm{Bu}_{4} \mathrm{~N}^{+}}=1$. Cette hypothèse conduit à attribuer aux ions étudiés les nombres de Hall notés $h_{+}$et $h_{-}$dans les tableaux I et II. On a alors $h_{+} \simeq 0,7$ pour $\mathrm{Cs}^{+}$et $\mathrm{K}^{+} ; h_{+}>0,7$ pour les autres cations $h_{-}<0,7$ avec $h_{\mathrm{Cl}^{-}}<h_{1^{-}}$dans le cas des solutions aqueuses. Dans le cas des solutions alcooliques, tous les nombres de Hall ioniques sont supérieurs à 0,7 et $h_{\mathrm{Cl}^{-}}>h_{\mathrm{I}^{-}}$.

Une autre hypothèse. doit être envisagée si on souhaite ne plus faire référence à l'ion $\mathrm{Bu}_{4} \mathrm{~N}^{+}$, peu inerte au point de vue de la solvatation, comme le remarque R. L. Kay [6], malgré sa taille relativement grande. On peut alors assimiler l'ion $\mathrm{Cs}^{+}$à une sphère rigide autour de laquelle les molécules de solvant de la première couche de solvatation peuvent tourner librement et poser $h_{\mathrm{Cs}^{+}}^{\prime}=0,7$, ce qui revient à admettre que le résultat prévu par Hubbart et Wolynes est exact pour cet ion. Les nombres de Hall notés $h_{+}^{\prime}$ et $h_{-}^{\prime}$ des tableaux I et II correspondent à cette deuxième hypothèse.

D'autres hypothèses sont encore possibles. On peut poser, par exemple, que dans l'eau $h_{\mathrm{Na}^{+}}^{\prime \prime}=0,7$, les résultats de la théorie de Hubbart et Wolynes étant supposés exacts pour cet ion dont " l'hydration ", d'après, par exemple, A. S. Faleev et al. [7] est pratiquement nulle. Compte tenu des erreurs expérimentales, les valeurs $h_{+}^{\prime \prime}$ et $h_{-}^{\prime \prime}$ ainsi obtenues dans le tableau I sont, à la limite, compatibles avec les valeurs $h_{+}, h_{-}$et $h_{+}^{\prime}, h_{-}^{\prime}$ correspondantes.

$\mathrm{Si}$, enfin, dans le méthanol, on admet que $h_{\mathrm{Cl}^{-}}=h_{\mathrm{I}^{-}}$, alors on obtient les valeurs notées $h_{+}^{\prime \prime}$ et $h_{-}^{\prime \prime}$ du tableau II, qui sont incompatibles avec les valeurs $h_{+}, h_{-}$et $h_{+}^{\prime}, h_{-}^{\prime}$ correspondantes.

4. Conclusion. - Les nombres de Hall ioniques sont liés à la dynamique des porteurs dans le solvant; comme l'a montré $H$. L. Friedman [8] ils sont sensiblement proportionnels au moment du premier ordre de la fonction d'autocorrélation temporelle de la vitesse :

$$
h_{\mathrm{i}} \simeq \frac{q_{\mathrm{i}}^{2}}{\mu_{\mathrm{i}}^{2} M_{\mathrm{i}}} \frac{1}{k T} \int_{0}^{\infty} t \mathrm{C}_{V_{x} V_{x}}^{0} \mathrm{~d} t
$$

$q_{\mathrm{i}}, \mu_{\mathrm{i}}$ et $\boldsymbol{M}_{\mathrm{i}}$ étant respectivement la charge, la mobilité et la masse de la particule.

Si $h=1$, le comportement de l'ion est, au moins en apparence, celui d'une particule brownienne : les interactions ion-solvant se font uniquement au cours de chocs. (Rappelons qu'une valeur de $h$ supérieure à l'unité pourrait correspondre, comme l'a suggéré H. L. Friedman [9], à la dynamique d'un «brownon à deux états ».)

Les différentes hypothèses de travail que nous avons envisagées ici donnent pour la plupart des ions étudiés des valeurs de $h$ nettement inférieures à un. Cela est particulièrement remarquable pour les ions chlorure et iodure en solution aqueuse.

Le cation $\mathrm{Li}^{+}$semble avoir dans les deux solvants un nombre de Hall relativement plus proche de l'unité que les cations $\mathrm{Cs}^{+}$et $\mathrm{K}^{+}$plus gros. Ce fạit peut être rapproché des résultats et conclusions des études effectuées par U. Kaatze et al. [10] relatives à la permittivité statique de solutions : il semble que l'ion $\mathrm{Li}^{+}$empêche la rotation des molécules de solvant de sa première couche de solvatation. Les interactions de l'ion et du solvant sont, en ce sens, des interactions fortes à courte portée. La dynamique de ce porteur se rapproche, au moins en apparence, de celle d'un brownon. 
Au contraire, les nombres de $\mathrm{Hall}$ faibles des anions $\mathrm{Cl}^{-}$et $\mathrm{I}^{-}$en solution aqueuse, font penser que la fonction d'autocorrélation des vitesses en fonction du temps de ces porteurs est tout d'abord très lentement décroissante, cela pendant un intervalle de temps non négligeable devant le temps de relaxation de la constante diélectrique $\tau_{\mathrm{D}}$, puis décroît ensuite très rapidement.

Il est remarquable que la dynamique de ces deux porteurs semble, suivant nos résultats, nettement différente dans l'eau et dans le méthanol.

\section{Bibliographie}

[1] Meton, M., Gérard, P., Chem. Phys. Lett. 44, number 3.

[2] Meton, M., Gérard, P., PiCard, E. J., J. Physique-Lett. 37 (1976) L-247-L-250.

[3] Abbes, M., Gérard, R., Gérard, P., Meton, M., PiCard, E. J., J. Physique-Lett. 41 (1980) L-575-L-580.

[4] Kay, R. L., Evans, D. F., J. Phys. Chem. 70 (1966) 2325.

[5] Hubbart, J. B., Wolynes, P. G., J. Chem. Phys. 75 (1981) 3051.

[6] Kay, R. L., Evans, D. F., Matesich, S. M., dans Solute-solvant interactions, Vol. 2, edited by J. F. Cootzee, C. D. Ritchie (1976), p. 117.

[7] Faleev, A. S., Kudryashova, V. A., Gaiduk, V. I., Rossukanyi, P. M. and Grigoriev, A. B., Russ. J. Phys. Chem. 51, 5 (1977).

[8] Harris, S., Friedman, H. L., J. Chem. Phys. 50 (1969) 765-770.

[9] Friedman, H. L., Ben-Naim, A., J. Chem. Phys. 48 (1968) 120-127.

[10] KaAtze, U., Adolph, D., Gottlob, D., Pottel, R., Ber. Bungenses. Phys. Chem. 84 (1980) 1198-1208. 